Results Among 168 patients with ASUC, 54 (32.1\%) required surgery and 114 (67.9\%) received intravenous corticosteroids or rescue treatment. CT scan showed 121 (72.0\%) patients had sarcopenia. Patients with sarcopenia had longer disease duration, more total parenteral nutrition, higher CRP level and lower quality of life. Although patients who required surgery had lower SMA, no significant differences were found in medical and surgical therapy between patients with and without sarcopenia. Patients with postoperative complications had higher preoperative white blood cell level and Creactive protein, and a higher incidence of sarcopenia. Multivariate analysis showed that sarcopenia (odds ratio, 53.07; 95\% confidence interval, 2.79-1010.03; $\mathrm{p}=0.008)$ was a negative predictor of postoperative complications in ASUC patients (table 1).

Abstract IDDF2019-ABS-0162 Table 1 Multivariate analyses of risk factors associated with postoperative complications for ASUC patients.

\begin{tabular}{llll}
\hline Characteristics & OR & $95 \% \mathrm{Cl}$ & P value \\
\hline Male & 1.163 & $0.232-5.835$ & 0.855 \\
Preoperative white blood cell & 17.666 & $2.036-153.274$ & 0.009 \\
Preoperative C-reactive protein & 2.456 & $0.259-23.310$ & 0.434 \\
Sarcopenia & 53.069 & $2.788-1010.032$ & 0.008 \\
\hline
\end{tabular}

ASUC, acute severe ulcerative colitis.

Conclusions The prevalence of sarcopenia was high in patients with ASUC. Sarcopenia was not a predictor of surgery, but a risk factor of postoperative complications in severe ulcerative colitis patients.

\section{IDDF2019-ABS-0163 PRELIMINARY RESULTS OF HIGH- RESOLUTION MANOMETRY IN DIAGNOSIS AND CLASSIFICATION FOR ACHALASIA}

${ }^{1}$ Linh Nguyen Thuy ${ }^{2}{ }^{2}$ Hang Dao Viet, ${ }^{1}$ Long Hoang Bao, ${ }^{2}$ Long Dao Van. ${ }^{1}$ IGH - Institute of Gastroenterology and Hepatology, Vietnam; ${ }^{2} \mathrm{HMU}$ - Hanoi Medical University, Vietnam

\subsection{6/gutjnl-2019-IDDFabstracts. 175}

Background Achalasia is a rare disease characterized by absent esophageal motility and disorder of lower esophageal sphincter (LES) relaxation. The Chicago Classification, version 3.0 presents the diagnostic criteria for achalasia using high-resolution manometry (HRM) as well as categorizes this disease into three types with different esophageal motility patterns.

Methods A case-series study was conducted at the Institute of Gastroenterology and Hepatology on patients suspected achalasia on upper endoscopy or esophageal barium X-ray. Symptom severity was evaluated by the Eckardt score before and after treatment. Achalasia was diagnosed and classified by HRM using the Chicago Classification version 3.0.

Results From April to December 2018, we recruited 20 patients $(7$ males and 13 females; the mean age were $35.9 \pm$ 15.4). There were $\underline{4}$ patients (20\%) diagnosed with absent contractility on HRM. In 16 achalasia confirmed patients, the percentage of type I, II, and III was $12.5 \%, 75 \%$, and $12.5 \%$, respectively. The mean Eckardt score before treatment was 6.6 \pm 2.6 and there was no difference between achalasia and absent contractility groups. The integrated relaxation pressure within $4 \mathrm{~s}$ (IRP4s) in achalasia group was high with the mean value being $24.6 \pm 6.3 \mathrm{mmHg}$ and there was no difference among three types. There was a significant improvement of clinical symptoms with pre and post-treatment Eckardt score being $6.8 \pm 2.8$ and $2.1 \pm 1.9$, respectively $(p<0.05)$. In 2 cases after surgery and balloon dilation, the LES pressure was normal but absent contractility and distal esophageal spasm were present on HRM.

Conclusions High-resolution manometry is a valuable exploratory test for definitive diagnosis, classification as well as follow-up after treatment on achalasia patients.

\section{IDDF2019-ABS-0164 THE MODIFIED PROGNOSTIC NUTRITIONAL INDEX (MPNI): A NEW RISK INDEX FOR PREDICTING CLINICAL OUTCOMES AFTER PERCUTANEOUS ENDOSCOPIC GASTROSTOMY}

Toh Yoon Ezekiel Wong*. Hiroshima Kyoritsu Hospital, Japan

\subsection{6/gutjnl-2019-IDDFabstracts.176}

Background Onodera's Prognostic Nutritional Index (O-PNI) is a screening tool derived from serum albumin levels (Alb) and total lymphocyte count (TLC). C-reactive protein (CRP) and blood urea nitrogen (BUN) levels were added to create the modified Prognostic Nutritional Index (mPNI). In this study, the utility of $\mathrm{mPNI}$ in predicting clinical outcomes after percutaneous endoscopic gastrostomy (PEG) was investigated.

Methods Based on multiple logistic regression analysis, mPNI was calculated using the equation $10+2 \mathrm{xAlb}(\mathrm{g} / \mathrm{dL})+\mathrm{TLC}($ $\mu \mathrm{L}) / 1000$ - $\mathrm{CRP}(\mathrm{mg} / \mathrm{dL}) / 2$ - BUN(mg/dL)/20. ROC analysis was used to evaluate $\mathrm{mPNI}$ as a predictor for postoperative aspiration pneumonia, in-hospital mortality, 30-day mortality and 90-day mortality.

Results

Derivation group 306 patients, mean age $82.6 \pm 10.0$ years old, Alb $3.2 \pm 0.5 \mathrm{~g} / \mathrm{dL}$, TLC $1357 \pm 586 / \mu \mathrm{L}$, CRP $2.5 \pm 2.9 \mathrm{~g} / \mathrm{dL}$, BUN $26.9 \pm 22.0 \mathrm{mg} / \mathrm{dL}, \mathrm{O}-\mathrm{PNI} 38.5 \pm 6.3$ and $\mathrm{mPNI} 15.1$ \pm 2.6 . Postoperatively, 69 patients developed aspiration pneumonia and 49 died before discharge, 22 within 30 days. AUC (area under the curve) for clinical outcomes summarized in table 1 .

\begin{tabular}{llll}
\multicolumn{4}{l}{ Abstract } \\
\hline & Aspiration pneumonia & In-hospital mortality & 30-day mortality \\
\hline Alb & 0.576 & 0.665 & 0.662 \\
TLC & 0.604 & 0.618 & 0.683 \\
CRP & 0.630 & 0.659 & 0.682 \\
BUN & 0.600 & 0.676 & 0.745 \\
O-PNI & 0.606 & 0.683 & 0.701 \\
mPNI & 0.680 & 0.759 & 0.801 \\
\hline
\end{tabular}

Validation group: 190 patients, mean age 81.4 18.6 years old, Alb $2.8 \pm 0.5 \mathrm{~g} / \mathrm{dL}$, TLC $1498 \pm 744 / \mu \mathrm{L}$, CRP $2.1 \pm 2.7 \mathrm{mg} /$ $\mathrm{dL}$, BUN $22.8 \pm 16.7 \mathrm{mg} / \mathrm{dL}$, O-PNI $35.7 \pm 6.8$ and $\mathrm{mPNI} 15.0$ \pm 2.5 . Postoperatively, 26 patients developed aspiration pneumonia, 19 died before discharge and 25 within 90 days. AUC for clinical outcomes summarized in table 2 . 


\section{Abstract IDDF2019-ABS-0164 Table 2}

\begin{tabular}{llll}
\hline & Aspiration pneumonia & In-hospital mortality & 90-day mortality \\
\hline Alb & 0.656 & 0.642 & 0.682 \\
TLC & 0.612 & 0.652 & 0.759 \\
CRP & 0.738 & 0.739 & 0.753 \\
BUN & 0.676 & 0.835 & 0.753 \\
O-PNI & 0.687 & 0.685 & 0.774 \\
mPNI & $\mathbf{0 . 7 5 4}$ & $\mathbf{0 . 8 2 2}$ & $\mathbf{0 . 8 5 0}$ \\
\hline
\end{tabular}

Conclusions The results of this study show that mPNI, an easy to calculate prognostic index using four biomarkers frequently measured during routine laboratory testing, is a strong predictor of both postoperative aspiration pneumonia and mortality after PEG.

\section{IDDF2019-ABS-0165 IMPACTS OF COEXISTING UPPER FUNCTIONAL GASTROINTESTINAL DISORDERS ON PSYCHOLOGICAL STATUS AND QUALITY OF LIFE IN IRRITABLE BOWEL SYNDROME}

${ }^{1}$ Xin Yao*, ${ }^{2}$ Yunsheng Yang, 'Shutian Zhang, ${ }^{3}$ Yu Shi. 'Department of Gastroenterology, Beijing Friendship Hospital, Capital Medical University, National Clinical Research Center for Digestive Disease, Beijing Digestive Disease Center, Beijing Key Laboratory for Precancerous Lesion of Digestive Disease, China; ${ }^{2}$ Institute of Digestive Diseases, Chinese PLA General Hospital, Chinese PLA Medical Academy, China; ${ }^{3}$ Clinical Epidemiology and EBM Unit, National Clinical Research Center for Digestive Disease, Beijing Friendship Hospital, Capital Medical University, China

\subsection{6/gutjn|-2019-IDDFabstracts. 177}

Background Functional dyspepsia (FD), belching disorders (BD) and functional heartburn (FH) were the three most frequent upper functional gastrointestinal disorders (FGID) in IBS patients. However, the impacts of concomitant FD, BD and FH on the health-related quality of life (HRQOL) and psychological status remain unclear.

Aims To investigate the impact of concomitant FD, BD and $\mathrm{FH}$ on the psychological problems and HRQOL in individuals with IBS.

Methods Consecutive outpatients with IBS were enrolled from 2 tertiary hospitals in China between January 2008 and March 2009. IBS, FD, BD and FH were diagnosed based on Rome III Criteria. Psychological status and HRQOL were evaluated using the validated Hospital Anxiety and Depression scale and 36-item Short-Form Health Survey (SF-36).

Results Of the 367 patients who met Rome III criteria for IBS, 319 patients agreed to participate in the study. These cases were further stratified into the subgroups of IBS+FD, $\mathrm{IBS}+\mathrm{BD} / \mathrm{FH}(\mathrm{BD}$ and/or $\mathrm{FH}), \mathrm{IBS}+\mathrm{FD}+\mathrm{BD} / \mathrm{FH}$, or IBS only according to the symptoms. IBS $+\mathrm{FD}$ group had significantly higher anxiety scores than IBS $+\mathrm{BD} / \mathrm{FH}$ group and greater depression than IBS only $(P<0.05)$. Multiple regression analysis showed that $\mathrm{IBS}+\mathrm{FD}+\mathrm{BD} / \mathrm{FH}$ was a predictive factor of worse mental component score (MCS), physical component score (PCS) and SF-36 global health while IBS+FD was a predictor of lower PCS and SF-36 compared with IBS only. Conclusions IBS patients overlapping with FD experience more anxiety, depression and lower PCS. The coexisting FD $+\mathrm{BD} / \mathrm{FH}$ is a predictive factor of reduced MCS and PCS in IBS patients.

\section{IDDF2019-ABS-0166 GASTROINTESTINAL STRESS SURVEY AND ENDOSCOPIC EVALUATION OF TROOPS IN HIGH ALTITUDE AREA}

${ }^{1}$ Du Shuwen* ${ }^{2}$ Wang Xin, ${ }^{3}$ Pan Yuanming, ${ }^{4} \mathrm{Xu}$ Junfeng, ${ }^{5} \mathrm{He}$ Yuqi, ${ }^{6} \mathrm{Kang}$ Qian, ${ }^{7}$ Wang Xiaoying, ${ }^{8}$ Sheng Jianqiu. ${ }^{1} D u$ shuwen The Seventh Medical Center of the General Hospital of the Chinese Peoplel's Liberation Army, China; ${ }^{2}$ Wang Xin The Seventh Medical Center of the General Hospital of the Chinese Peoplel's Liberation Army, China; ${ }^{3}$ Pan Yuanming The Seventh Medical Center of the General Hospital of the Chinese Peoplel's Liberation Army, China; ${ }^{4} X$ u Junfeng Peoplel's Liberation Army General Hospital, PLA Medical College, China; ${ }^{5} \mathrm{He}$ Yuqi The Seventh Medical Center of the General Hospital of the Chinese Peoplel's Liberation Army, China; ${ }^{6}$ Kang Qian The Seventh Medical Center of the General Hospital of the Chinese Peoplel's Liberation Army, China; ${ }^{7}$ Wang Xiaoying The Seventh Medical Center of the General Hospital of the Chinese Peoplel's Liberation Army, China; ${ }^{8}$ Sheng Jianqiu The Seventh Medical Center of the General Hospital of the Chinese Peoplel's Liberation Army, China

\subsection{6/gutjnl-2019-IDDFabstracts. 178}

Background To understand the incidence of gastrointestinal stress response and related physical and chemical indicators and endoscopic mucosal evaluation of troops in high altitude areas.

Methods A total of 614 troops were drawn from the area of golmud (377 with an average altitude of $3000 \mathrm{~m}$ ) and yushu (237 with an average altitude of $4,200 \mathrm{~m}$ ), The epidemiological investigation was conducted by self-made questionnaire of gastroenterology epidemiology of troops. The serum helicobacter pylori antibody and fecal occult blood were detected in the field, and the correlation between gastrointestinal symptoms and $\mathrm{Hp}$ infection and fecal occult blood was analyzed, and portable integrated digestive endoscopy was performed on soldiers with gastrointestinal stress reaction to evaluate the status of mucosa under the endoscope.

Results The incidence of gastrointestinal stress was as high as 50.8\%(312/614), including diarrhea 38.4\% (236/614), anorexia $35 \%$ (215/614), abdominal distension $27.9 \% \quad(171 / 614)$, abdominal pain $17.6 \%$ (108/614), halitosis $17.6 \%(108 / 614)$, hematemesis 7.3\% (45/614). Among them, we conducted gastroscopy on 212 of soldiers with gastrointestinal stress symptoms and found that all soldiers suffered from different degrees of gastroesophageal and duodenal inflammatory changes, most of which were gastritis, ulcer and mucosal erosion and bleeding.

Conclusions The incidence of gastrointestinal stress in highaltitude troops is high, mainly diarrhea, abdominal distension, abdominal pain, constipation. The main manifestations under gastroscope are gastritis, acute and chronic gastric mucosal erosion, duodenal ulcer. We should actively explore prevention and control measures for high altitude gastrointestinal stress to improve the gastrointestinal health of troops and improve the combat capability of troops in the plateau environment. 